\title{
Tunable free-electron X-ray radiation from van der Waals materials
}

Shentcis, Michael; Budniak, Adam K.; Shi, Xihang; Dahan, Raphael; Kurman, Yaniv; Kalina, Michael; Herzig Sheinfux, Hanan; Blei, Mark; Svendsen, Mark Kamper; Amouyal, Yaron

Total number of authors:

17

Published in:

Nature Photonics

Link to article, DOI:

$10.1038 / \mathrm{s} 41566-020-0689-7$

Publication date:

2020

Document Version

Early version, also known as pre-print

Link back to DTU Orbit

Citation (APA):

Shentcis, M., Budniak, A. K., Shi, X., Dahan, R., Kurman, Y., Kalina, M., Herzig Sheinfux, H., Blei, M.,

Svendsen, M. K., Amouyal, Y., Tongay, S., Thygesen, K. S., Koppens, F. H. L., Lifshitz, E., García de Abajo, F. J., Wong, L. J., \& Kaminer, I. (2020). Tunable free-electron X-ray radiation from van der Waals materials. Nature Photonics, 14(11), 686-692. https://doi.org/10.1038/s41566-020-0689-7

\section{General rights}

Copyright and moral rights for the publications made accessible in the public portal are retained by the authors and/or other copyright owners and it is a condition of accessing publications that users recognise and abide by the legal requirements associated with these rights.

- Users may download and print one copy of any publication from the public portal for the purpose of private study or research.

- You may not further distribute the material or use it for any profit-making activity or commercial gain

- You may freely distribute the URL identifying the publication in the public portal 


\title{
Tunable free-electron X-ray radiation from van der Waals materials
}

\author{
Michael Shentcis', Adam K. Budniak², Xihang Shi', Raphael Dahan', Yaniv Kurman', \\ Michael Kalina ${ }^{3}$, Hanan Herzig Sheinfux ${ }^{4,5}$, Mark Blei ${ }^{6}$, Mark Kamper Svendsen ${ }^{7}$, Yaron \\ Amouyal $^{3}$, Sefaattin Tongay ${ }^{6}$, Kristian Sommer Thygesen ${ }^{7}$, Frank. H.L. Koppens ${ }^{4,5}$, Efrat \\ Lifshitz $^{2}$, F. Javier García de Abajo ${ }^{4,5}$, Liang Jie Wong ${ }^{8}$ and Ido Kaminer ${ }^{1}$ \\ ${ }^{1}$ Department of Electrical Engineering, Technion - Israel Institute of Technology, Haifa
} 3200003, Israel

${ }^{2}$ Schulich Faculty of Chemistry, Technion - Israel Institute of Technology, Haifa 3200003, Israel

${ }^{3}$ Department of Materials Science and Engineering, Technion - Israel Institute of Technology, Haifa 3200003, Israel

${ }^{4}$ ICFO - Institut de Ciencies Fotoniques, The Barcelona Institute of Science and Technology, 08860 Castelldefels (Barcelona), Barcelona, Spain

${ }^{5}$ ICREA - Institució Catalana de Recerca i Estudis Avançats, Passeig Lluís Companys 23, 08010 Barcelona, Spain

${ }^{6}$ School for Engineering of Matter, Transport and Energy, Arizona State University, Tempe, Arizona 85287, USA

${ }^{7}$ CAMD, Department of Physics, Technical University of Denmark, DK-2800 Kongens Lyngby, Denmark

${ }^{8}$ School of Electrical and Electronic Engineering, Nanyang Technological University, 50

Nanyang Ave, Singapore 639798, Singapore 
Tunable sources of $X$-ray radiation are widely used for imaging and spectroscopy in fundamental science, medicine, and industry. The growing demand for highly tunable, high-brightness lab-scale X-ray sources motivates research into new fundamental mechanisms of X-ray generation. Here, we demonstrate the ability of van der Waals (vdW) materials to serve as a platform for tunable X-ray generation when irradiated by moderately relativistic electrons available, for example, from a transmission electron microscope (TEM). The radiation spectrum can be precisely controlled by tuning the acceleration voltage of the incident electrons, and also by a newly introduced approach adjusting the lattice structure of the vdW material. We present experimental results for both methods, observing energy tunability of X-ray radiation from the vdW materials $\mathrm{WSe}_{2}, \mathrm{CrPS}_{4}, \mathrm{MnPS} 3, \mathrm{FePS}_{3}, \mathrm{CoPS}_{3}$ and NiPS 3 . Our findings demonstrate the concept of material design at the atomic level, using vdW heterostructures and other atomic superlattices, for exploring novel phenomena of X-ray physics. 


\section{Introduction}

The wealth of unique properties of van der Waals (vdW) materials in either bulk or single-atomic-layer form have constituted the basis of many novel physical phenomena and fundamental advances in recent years [1,3]. For example, graphene, an atom-thick layer of graphite [4], exhibits ultrahigh carrier mobility at room temperature [5], excellent optical transparency [6], high Young's modulus [7], high thermal conductivity [8] and many other properties of practical utility. Moreover, this material has enabled the observation of novel phenomena such as the room temperature quantum Hall effect [9]. Transition metal dichalcogenides (TMDs) [10,11] and transition metal thiophosphates (TMTs) [12] have also emerged as intriguing families of $\mathrm{vdW}$ materials. Semiconductors by nature, TMDs can exhibit both indirect band-gap in the bulk or direct band-gap as a single-atomic layer [10]. This unique property, combined with weak dielectric screening in two dimensions [13], gives rise to strong photoluminescence and large exciton binding energies, making TMDs attractive materials for light emitting devices [14]. Likewise, TMTs are layered semiconductors additionally characterized by unique magnetic properties that make them potentially useful for application in quantum information devices and in spintronics [15].

Here, we show how vdW materials can be used to explore novel phenomena of X-ray physics, in which free electrons passing through vdW layered structures create tunable X-ray radiation. Our findings thus present a new class of applications for vdW materials, as possible platforms for X-ray sources with table-top footprint that have advantages in being tunable and monochromatic relative to all existing compact sources. We demonstrate the unprecedented tunability of X-ray generation using free electrons passing through vdW materials and explore the processes by which the X-ray is generated with both theory and experiments (Fig. 1a-c). The 
two mechanisms involved are parametric X-ray radiation (PXR) and coherent bremsstrahlung (CBS) [16-19]. Both mechanisms arise simultaneously as a result of periodic interactions of free electrons propagating through an atomic structure (more detailed descriptions are provided below).

In our experiment, the output photon energy is controlled by changing the incident electron energy, as well as through a new method based on adjusting the composition and stacking of the vdW material structure. We present a comparative study demonstrating the highly precise dependence of the X-ray energy spectrum on the choice of the transition metal atom in the vdW material (Fe, Co or Ni in XPS3). Looking at the big picture, the experimental results presented constitute a proof-of-principle for a wider designer approach that we propose: to precisely tailor the radiation energy spectrum and angular distribution of X-ray emission by means of material design at the atomic level. We show that a wide range of superlattice crystalline materials, both natural and artificial, can be used for X-ray generation and we present a theory for X-ray emission from designed superlattice structures. These findings pave the way toward the use of superlattice atomic structures for the realization of tunable and versatile sources of X-ray radiation with a table-top footprint.

The appeal of using vdW materials for X-ray generation is enhanced by the fact that many vdW materials possess high in-plane thermal conductivities [20] and some have higher melting temperatures compared to conventional materials. Moreover, radiation damage can be further reduced by using heterostructures combining different kinds of vdW materials [21, 22]. Other qualities are specifically attractive for PXR and CBS and arise from the weak bonding between the layers in vdW materials, which helps maintain the crystalline nature of the material 
that is crucial for both mechanisms. Moreover, the crystal structures of vdW materials are characterized by larger unit cells compared to those of conventional 3D bulk crystalline materials. As showed below, the larger unit cell enables X-ray generation with relatively high brightness in spectral ranges such as the water window (useful for biological imaging [23]), using electron energies obtainable from lab-scale electron sources such as the ones used in transmission and scanning electron microscopes (TEMs and SEMs). Furthermore, the wide range of compositions and flexibility in the stacking of $\mathrm{vdW}$ materials provides extra tunability to shape the output radiation through the control of the atomic lattice geometry.

The energy tunability of X-ray sources is a key factor in many applications such as core level spectroscopy [24-26] and various X-ray imaging techniques [27-29]. The energy tunability required for such applications is usually achieved in undulator facilities, such as synchrotrons, and free-electron lasers [30-32]. However, the operation of such facilities necessitates immense resources in terms of space, energy and safety measures, which limit their accessibility. Most lab-scale sources take the form of the X-ray tube, in which free electrons are used to induce bremsstrahlung or core transitions that produce X-rays. Such sources do not provide energy tunability or radiation directionality capabilities, which have a lot of potential for a wide variety of uses.

These limitations motivate the research of new physical mechanisms for X-ray generation that may have a potential to create lab-scale X-ray sources that are tunable and directional. In particular, research advances of X-ray generation mechanisms led to developments of laserdriven Compton-based X-ray sources [33] that utilize the micron-scale periodicity of light, which is much smaller than the millimeter/centimeter scale of conventional undulator facilities. Such smaller scale undulators enable reducing the levels of electron acceleration, while still resulting 
in tunable X-ray generation. More recent proposals rely on shrinking the undulating periodicity even further by leveraging the high electromagnetic confinement in graphene surface plasmons [34], metasurfaces [35], metamaterials [36] and nanophotonic vacuum fluctuations [37]. Our study explores different phenomena that lead to X-ray generation from free electrons, in which the electron undulation is done at the ultimate periodicity: that of the atomic crystal lattice.

\section{Results: Tunable X-Ray Radiation from Van der Waals Materials}

The vdW-material-based atomic undulator that we present here relies on two radiation mechanisms, CBS and PXR, using modest electron energies that are available in relatively simple systems such as a TEM. The mechanisms of CBS and PXR have not yet been studied in $\mathrm{vdW}$ materials. Both mechanisms take place as a propagating electron undergoes coherent interaction with the intrinsic periodicity of a crystalline material (Fig. 1).

PXR is emitted from the modulation induced by the incident electron on the bound electrons of the material's atoms, creating polarization currents from which directional X-ray radiation is emitted. The PXR mechanism can also be described as diffraction of the incident electron's Coulomb field off the periodical atomic arrangement of the structure (Fig. 1b). We model PXR by treating the periodic atomic structure as a discrete dipole array, with bound electrons around each atom being modelled as an effective dipole quantified through an associated X-ray atomic polarizability.

CBS is emitted from the incident electron as a result of a periodic series of bremsstrahlung interactions with the nuclei and bound electrons in the material, where each interaction involves the acceleration of the electron and causes it to emit radiation (Fig. 1c). The modulation of the free-electron velocity by the periodic potential results in interference of the 
emitted radiation and causes it to become directional. We model CBS by considering the farfield radiation from electrons whose trajectories are calculated by using the relativistic NewtonLorentz equations in the presence of an atomic potential, which is in turn obtained from density functional theory (see Methods).

The radiation emitted from each electron through the CBS and PXR mechanisms maintains temporal and spatial coherence (in contrast to coherence from multiple electrons which requires bunching of the electrons). i.e., the radiation emitted from multiple locations along the trajectory of the incident electron interferes constructively to produce angle-dependent output radiation (the coherent interference from the multiple locations is possible because the final quantum state of the material is not changed). The radiation from both mechanisms follows the same dispersion relation [19],

$$
E_{m}=\hbar \omega_{m}=m \frac{2 \pi \hbar c \beta \cos (\theta)}{d[1-\beta \cos (\varphi)]}, \quad m=1,2,3 \ldots,
$$

where $\beta=v / c$ is the normalized speed of the electron; $E_{m}$ and $\omega_{m}$ are the photon energy and angular frequency, respectively, corresponding to radiation of order $m$; $\hbar$ is the reduced Planck constant; $\mathrm{d}$ is the lattice constant; and $\theta$ and $\varphi$ are the angles defining the electron velocity relative to the reciprocal lattice of the crystal and the photon emission direction, respectively.

Each of the PXR and CBS mechanisms becomes of greater significance at different regimes of emission energies and angles. However, they both share the same dispersion relation (Eq. 1), and thus they can be effectively considered as a single combined effect [38,39], which we shall refer to as parametric coherent bremsstrahlung (PCB) in the remainder of the manuscript. 
The unique structure and large variety of vdW materials make them promising candidates for PCB radiation for several reasons. One reason arises from the unique layered-structure of $\mathrm{vdW}$ materials that are made of strong covalently bonded atomic layers joined together by vdW forces. Consequently, the effect of integrating certain atoms (e.g., W) into a vdW structure (e.g., WSe2) can be seen as effectively "stretching" the lattice constant $d$, thus redshifting the radiation energy peaks $E_{\mathrm{m}}$. For example, the lattice constant of bulk W, $3.16 \AA$ is increased to $12.98 \AA$ (unit cell size in an A-B stacking, i.e., distance between two alternate-layers) when it is embedded into a WSe2 TMD structure, giving rise to PCB X-ray radiation with a different energy-angle profile for the same incident electron velocities (Fig. 1d). The longer effective period of the vdW crystal allows soft X-ray photons (e.g., in the water window) to be created with higher incident electron energy than in regular 3D bulk crystals - seen by the tradeoff of $d$ and $\beta$ in Eq. 1. This regime of parameters leads to higher output power and brightness using electrons from TEMs and SEMs.

Figures $2 \mathrm{a}$ and $2 \mathrm{~b}$ present $\mathrm{PCB}$ radiation from additional vdW structures, namely, MnPS3 and CrPS4, oriented along the [103] and [001] zone axes respectively. As in Fig. 1d, we note a good agreement of the experimental results with the predicted energy values calculated from Eq. 1.

Using the PCB theory, we also predict the width $\Delta \omega$ of the spectral peaks, which for thin TEM samples and highly directional electron beams yields (Supplementary Section S.1),

$$
\frac{\Delta \omega}{\omega_{m}}=\frac{\Delta E}{E_{m}} \approx \sqrt{0.8 \frac{d^{2}}{m^{2} L^{2}}+\Delta \varphi_{\mathrm{D}}^{2} \frac{\beta^{2} \sin ^{2} \varphi}{[1-\beta \cos \varphi]^{2}}+\Delta \theta_{\mathrm{e}}^{2} \tan ^{2} \theta}
$$

with $\mathrm{L}$ being the electron interaction length in the crystal, $\Delta \varphi_{\mathrm{D}}$ the angular aperture of the detector and $\Delta \theta_{\mathrm{e}}$ the angular spread of the incident electron beam. In the present study, the width 
of the spectral lines is primarily determined by the angular aperture of the energy-dispersive Xray spectrometer (EDS), $\Delta \varphi_{\mathrm{D}}=16^{\circ}$, which collects the emission for a range of angles $\varphi=$ $113^{\circ}-129^{\circ}$. In contrast, the left term in the square root of Eq. 2 is comparatively negligible in our case because the crystal thicknesses of the different materials $(\mathrm{L} \sim 100 \mathrm{~nm})$ do not introduce significant broadening relative to the angular aperture of the detector. Similarly, $\Delta \theta_{\mathrm{e}}<0.1 \mathrm{mrad}$, estimated to be the electron beam divergence angle in our experiment, is small and does not significantly alter $\Delta \omega$. An additional effect of significant broadening is the detector energy resolution $(\sim 80 \mathrm{eV})$, which has to be combined with the result of Eq. 2 (see Methods).

We can use Eq. 2 to estimate the monochromaticity of the emitted radiation, in a way that is independent of the detector parameters. Neglecting $\Delta \varphi_{\mathrm{D}}$ in it, we find that PCB radiation generated by a collimated electron beam results in $\Delta \omega / \omega_{\mathrm{m}}=0.9 \mathrm{~d} / \mathrm{mL}$, which means $\frac{1.2}{\mathrm{~m}} \%$ for interaction length of $100 \mathrm{~nm}$ WSe2. Therefore, when collected over a small angle, the X-rays produced are indeed very monochromatic, possessing a narrow bandwidth below $1 \%$ for orders $m \geq 2$.

The positions of the experimental peak maxima in Figs. 1d and 2a-c show small deviations from the theoretical values (marked by vertical dashed lines), possibly due to small uncertainties in the detector angle, or in the reported values of the interlayer distances used here as input parameters. Additional effects that explain deviations between theory and experiment include the background of incoherent bremsstrahlung radiation, different interaction lengths for each incident electron energy, divergence of the incident electron beam, and further subsequent diffraction of the radiation by the crystal and the detector (Supplementary Section S.7). The latter should be sensitive to the properties of the electron beam because it essentially consists of Kossel-line-like diffraction from a coherent source made of the emission emanating from all of 
the atoms exposed to each incident electron wave. Additionally, our PXR simulation shows that since the detector is located in the backward direction relative to the electron velocity, there is a small Doppler shift that causes the energy peak to be slightly redshifted, in qualitative agreement with our measurements (seen in the theory and experiments in Fig. 1d).

We use the simulation tools here developed for CBS and for PXR to quantify both mechanisms and compare them with our experimental results. We find that in our regime of interest (moderately relativistic electrons available from TEMs), the PXR mechanism dominates the radiation emission as it is two orders of magnitude larger than CBS. These models enable the average brightness values of PCB radiation sources from vdW materials to be theoretically predicted. For example, we examine the brightness values obtainable in a TEM setup such as used in this work: A relatively low electron current of $\sim 1 \mathrm{nA}$ and a $\sim 1 \mathrm{~nm}$ electron beam diameter (at the plane of the sample) passing through a WSe2 sample of $\sim 100 \mathrm{~nm}$ thickness. For the detector orientation of $\varphi=121^{\circ}$ with respect to the electron velocity, we estimate a brightness value of $\sim 1 \cdot 10^{9} \frac{\text { photons }}{\mathrm{s} \cdot \mathrm{mrad}^{2} \cdot \mathrm{mm}^{2} \cdot 0.1 \% \mathrm{BW}}$ within the range of energy tunability that we show of $\sim 700$ $1100 \mathrm{eV}$. This level of brightness compares favorably with state-of-the-art X-ray tubes, while the input power is smaller by a factor of $10^{-5}-10^{-8}$ (because the electron spot diameter in our experiment is much smaller than that of X-ray tubes). Moreover, our PCB radiation is directional and tunable in contrast to the radiation from X-ray tubes (which is either characteristic or bremsstrahlung). The estimated number of photons detected at the detector's solid angle: $4 \times$ $10^{3}, 2.6 \times 10^{3}, 1.8 \times 10^{3}, 1.5 \times 10^{3}, 1.4 \times 10^{3}, 1.1 \times 10^{3}, 1.0 \times 10^{3}$ photons $/(\mathrm{s} \cdot \mathrm{eV})$ at each peak from $60 \mathrm{keV}$ to $300 \mathrm{keV}$ in Fig. 1d, respectively (details in Methods). The typical number of photons per second is $\sim 10^{5}$ (integrating over the widths of the peaks). 
The brightness of the PCB source can be further improved by optimizing parameters such as the detector orientation and size, the electron acceleration voltage, and the sample thickness (similarly to other undulation-based emission mechanisms, the PCB brightness is quadratic with the interaction length). It can also be increased by using larger currents and reducing the electron spot size, for which an optimum compromise can be found by keeping in mind the tradeoff enforced by Coulomb repulsion (space charge), which leads to greater beam divergence (hence smaller interaction length) with larger electron density. When the electron beam divergence exceeds about 0.1 degrees, the heights of the radiation peaks start to broaden and decrease significantly ( $>5 \%$ from their original values). When considering space charge, we show in Fig. S5 (in Supplementary Section S.5) that the potential increase in brightness of PCB from vdW materials can be as much as $10^{7}$ times the value reported above (for an optimal combination of beam current and beam size).

The X-ray generation from the $\mathrm{PCB}$ mechanism can be extended to the hard X-ray regime with higher electron energies, as we show in Supplementary Section S.4, where we simulate the output radiation from $1 \mathrm{MeV}$ and $5 \mathrm{MeV}$ electrons. Electron beams generated by relatively compact sources such as photoemission injectors based on RF guns and DC high voltage guns $[40,41]$ enable higher electron currents of up to tens of $\mathrm{mA}$ in acceleration voltages of a few $\mathrm{MeV}$, which can strongly improve the emission brightness from thicker crystal samples.

The performance of PCB radiation from vdW materials can be also viewed from the perspective of energy transfer and efficiency. The total probability of each $60 \mathrm{keV}$ electron going through a $100 \mathrm{~nm}$ interaction length in WSe2 to produce $\mathrm{PCB}$ emission is $\sim 1 \cdot 10^{-4}$, resulting in an average electron energy loss to PCB of $0.25 \mathrm{eV}$. Out of this, the probability of radiation in the direction of the detector is $\sim 1 \cdot 10^{-5}$, i.e., electron energy loss to "useful X-ray photons" of 
$0.025 \mathrm{eV}$. This probability is on the same order of magnitude as is found in related processes such as Smith-Purcell radiation. The efficiency can be further improved because the electron energy can be reused. Such an approach is used in many free-electron-based applications such as traveling wave tubes. Recycling the electron energy means that the absolute efficiency depends on competing channels of energy loss in the sample. In the case of PCB radiation, such processes mainly come from Coulomb collisions that result in the ionization of other electrons, excitation of atoms and bremsstrahlung radiation. We use a numerical simulator developed by NIST to estimate the total energy loss of a $60 \mathrm{keV}$ electron in a $100 \mathrm{~nm}$ sample from the materials we use, and find an average energy loss of $\sim 300 \mathrm{eV}$. Therefore, we predicted the efficiency of the mechanism to be $\sim 0.1 \%$ ( $60 \mathrm{keV}$ electrons, $100 \mathrm{~nm}$ WSe2).

At higher energies, the efficiency improves as the radiation is more directional due to relativistic contraction. In addition, higher electron energies also have longer penetration depths in thicker samples (i.e., lower competing loss channels). Overall, such efficiency can be tolerable when considering that X-ray sources are never generally efficient and keeping in mind that, unlike any other compact X-ray source, PCB based sources are tunable.

\section{Discussion: Designer Superlattice X-Ray Sources}

We now discuss methods to control the emitted spectra from PCB radiation. The X-ray energy is tuned in Figures 1d, 2a and $2 b$ by adjusting the incident electron energy. However, such a method necessitates a realignment process to ensure that different conditions, such as the quality of the electron beam collimation, remain unchanged, thus adding a degree of difficulty to that approach. Another method for adjusting the output photon profile involves changing the orientation of the crystal with respect to the electron beam, which unfortunately compromises the emission efficiency and other properties of the output radiation. 
Taking a different approach, we present in Fig. 2c a new method for controlling the output radiation spectra by precise manipulation of the lattice structure. Using several different $\mathrm{vdW}$ materials with similar basic structure in which the main element is altered, we show that the resulting small but precise changes in the lattice constant lead to a corresponding precision control over the spectral peaks of the emitted PCB radiation. Figure $2 \mathrm{c}$ shows three vdW materials sharing the formula XPS3, where $\mathrm{X}$ represents the transition metals $\mathrm{Fe} / \mathrm{Co} / \mathrm{Ni}$. The lattice constant differs slightly between these vdW compounds (see Fig. 2c, right inset), enabling fine energy tuning of the emission through lattice variation. This tuning method provides an extra handle of versatility on top of the conventional PCB tuning mechanisms of electron energy and orientation, further supporting the potential of vdW materials as compact platforms for highquality X-ray radiation generation.

Tunability by structural design of $\mathrm{vdW}$ materials is the first step towards the broader designer concept that we propose, in which we can use a specially engineered superlattice structure for spectral shaping of the emitted X-ray radiation. Analogous structural customizations for manipulation of radiation were suggested for other applications at optical wavelengths in metasurfaces [42] and in designer Smith-Purcell gratings [43,44]. However, this approach has never been considered for X-ray radiation manipulation.

Figure $3 \mathrm{a}$ presents our designer approach by exploring a general superlattice configuration in which two crystalline materials are combined into a superlattice. Mathematically, we derive a corrected dispersion relation where the lattice factor $\mathrm{d}$ in Eq. 1 is replaced by a weighted sum of lattice factors $d_{1} n_{1}+d_{2} n_{2}$, with $d_{1}, d_{2}$ denoting the lattice constants and $\mathrm{n}_{1}, \mathrm{n}_{2}$ the number of layers in each period of the two combined crystals respectively. We thus have (generalizing Eq. 1): 


$$
E_{m}^{\prime}=\hbar \omega_{m}^{\prime}=m \frac{2 \pi \hbar \beta \cos \theta}{\left(d_{1} n_{1}+d_{2} n_{2}\right)(1-\beta \cos \varphi)}, m=1,2,3 \ldots
$$

PCB radiation from monocrystalline materials is usually strongest for the 1st emission order $(m=1)$. However, in superlattices made up of two materials, the dominant emission would occur at the order $m=n_{1}+n_{2}$ (i.e., the total number of layers in a single superlattice period).

Figures $3 \mathrm{~b}$ and $3 \mathrm{c}$ show that changing the atomic composition of the superlattice period allows us to control the range of emitted radiation energy for the same incident electron velocities. The curves in Fig. $3 \mathrm{~b}$ are calculated by combining graphite $\left(\mathrm{d}_{1}=0.3308 \mathrm{~nm}\right)$ and hexagonal boron nitride $\left(\mathrm{d}_{2}=0.3350 \mathrm{~nm}\right)$ into a superlattice structure and presenting several emission orders of such structures. The theoretical estimate of the intensity relation between emission orders in Fig. 3c is based on a model that uses a one-dimensional array of radiating dipoles. In this model, each dipole represents a lattice plane of the superlattice. As the PCB energy profile is dependent on the constructive interference of emission from consecutive lattice planes, we can calculate the relation between the emission peaks by considering the electromagnetic field emitted from a row of point dipoles with the corresponding relative phases. We note an excellent match between the emitted energies presented in this model and the dispersion relation in Eq. 3.

\section{Outlook}

We envision the use of $\mathrm{vdW}$ heterostructures $[45,46]$ and other artificial superlattice crystalline materials to optimize the emission of PCB X-ray radiation with user-defined spectral and spatial properties. These sources are also attractive due to practical considerations, such as the promise of higher stability and smaller dependence on recalibrations and electron beam 
alignments, because it is possible to tune the emission by changing between a series of superlattices without modifying the electron beam properties. Fabrication of such superlatticePCB sources can benefit from a variety of new crystal growth techniques, as well as the large wealth of possible chemical compositions and crystal structures offered by vdW materials. Additionally, molecular beam epitaxy (MBE) [47], metal organic chemical vapor deposition (MOCVD) [48] and other established material-growth techniques could be used to enable superlattice design incorporating other material families (e.g., III-Vs, II-VIs, etc.) for X-ray radiation generation and manipulation.

Another significant improvement would be the use of nano-modulated electrons that could make the radiation coherent from multiple electrons (microbunching). These nanomodulated electrons can be generated via emittance exchange techniques [49,50], laser-plasma interactions [51], or electromagnetic intensity gratings [52]. Future work could develop structures that achieve resonant PCB conditions for the interaction between the propagating electrons and the periodic structure in order to improve the emission efficiency of PCB radiation.

Our theoretical and experimental results show the advantages of vdW materials in engineering the emission of energy-tunable X-ray radiation. VdW materials add versatility to Xray energy tuning of PCB radiation based on a novel lattice modification technique that can be combined with previously-explored tuning techniques or used as a separate method. Additionally, this novel technique demonstrates the advantages of designer superlattices for energy tunable emission of monochromatic and directional X-ray radiation.

In contrast to state-of-the-art tunable X-ray sources, PCB radiation can be generated from modestly relativistic electrons. This makes the superlattice-PCB X-ray sources relatively 
compact and more accessible for various applications such as X-ray spectroscopy [24-26]. For example, PCB radiation from vdW materials can be used for compact and tunable X-ray photoemission spectroscopy (XPS) methods. Such an application is already within reach using the emission flux and brightness of PCB from the low current of a basic TEM setup, as described above.

Additional research tools such as transmission X-ray microscopy and scanning photoelectron microscopy [53] are also potential applications for future PCB sources. X-ray imaging techniques can benefit from the use of energy-tunable sources, as the image contrast is affected by the energy of the incident radiation [27-29]. This concept may be used, for instance, to improve the yield and accuracy of mammography techniques, in addition to reducing the radiation dose delivered to a patient [28]. Future imaging techniques that use the tunability of superlattice-PCB X-ray sources can create compact variants of K-edge imaging techniques [54] with limited brightness and flux, yet at accessible lab-scales.

In conclusion, we theoretically predict and experimentally demonstrate tunable, highbrightness X-ray emission from vdW materials when irradiated by moderately relativistic electrons available, for example, from a TEM. The radiation spectrum can be precisely controlled by tuning the acceleration voltage of the incident electrons, and also by a newly introduced approach - adjusting the lattice structure of the vdW material. We present experimental results for both methods, observing energy tunability of X-ray radiation from the vdW materials WSe2, CrPS4, MnPS3, FePS3, CoPS3 and NiPS3. Our findings demonstrate the concept of material design at the atomic level, using vdW heterostructures and other atomic superlattices, for exploring novel phenomena of X-ray physics. Looking forward, the control of atomic layers in both the longitudinal and transverse directions would enable spatial, angular and 
spectral shaping of output X-ray radiation. We envision the customization of superlattices for user-specific applications via inverse design techniques that optimize desired output radiation characteristics given the electron beam conditions and the specific geometrical constraints. 


\section{Main Figure Legends}

Figure 1: Demonstration of free-electron radiation from van der Waals (vdW) materials. (a) The radiation is produced from two combined mechanisms (termed PCB collectively): parametric X-ray radiation (PXR) and coherent bremsstrahlung (CBS), both emitted with the same dispersion relation (Eq. 1) from an electron propagating through periodic crystal structures. (b) Illustration of the PXR mechanism, produced as the particle's electromagnetic field (light blue halo) is diffracted off the periodic crystal structure. (c) Illustration of the CBS mechanism, describing the X-ray emission by an undulated electron due to a series of periodic bremsstrahlung interactions with the crystal lattice. (d) Photon energy spectrum of X-ray radiation created by an electron moving along the [001] zone axis of $\mathrm{WSe}_{2}$. The emitted radiation is peaked at different photon energies depending on the electron kinetic energy $(60-300 \mathrm{keV})$. The experimental results (dotted curves) are in good agreement both with the theoretical prediction for the peak energy (Eq. 1 with $\mathrm{m}=2$, vertical dotted lines) and with the peak width (Eq. 2, horizontal dashed lines) using no fitting parameters. We compare the results with simulations of PXR and CBS and present the PXR theory that is found to be stronger in this case (solid curves). The trends match the experimental observation with the only fitting parameters being the y-axis scaling of each curve. We attribute deviations of the experimental results from the simulated curves to emission from core transitions (a broad peak around $\sim 780 \mathrm{eV}$ and on the right edge). Insets: Sample image and diffraction pattern (top right); 3D model (bottom right).

Figure 2: Tunability of X-ray radiation from vdW materials. (a,b) X-ray radiation spectra collected from $\mathrm{MnPS}_{3}$ (a) and $\mathrm{CrPS}_{4}$ (b). The photon energy peak is tuned by varying the incident electron kinetic energy in the range of $60-300 \mathrm{keV}$, as indicated in the panels. Theoretically predicted peak energy values (Eq. 1 for $m=2$, dotted vertical lines) and energy peak widths (Eq. 2 horizontal dashed lines) both show a good match with experimental results. The constant energy peak in (b) is the characteristic radiation peak emitted from copper (marked as "Cu").The insets present images and diffraction patterns of each vdW material for [001] $\left(\mathrm{CrPS}_{4}\right)$ and [103] $\left(\mathrm{MnPS}_{3}\right)$ crystal orientations, as well as 3D models of the layered vdW structures. (c) Radiation from three vdW materials with the same crystal structure $\left(\mathrm{FePS}_{3}\right.$ (purple), $\mathrm{CoPS}_{3}$ (blue), and $\mathrm{NiPS}_{3}$ (green)), but differing by a picometer-scale difference in the lattice constant $d$. The resulting radiation energy tuning through structural modification is demonstrated at two different incident electron kinetic energies, showing the possibility for a combined X-ray energy tunability via variation of the structure and of the electron acceleration voltage. The sensitivity of the photon-energy tuning is high, being only limited by the energy resolution and angular aperture of the detector.

Figure 3: Spectral shaping of $\mathrm{X}$-ray radiation via customized superlattices. (a) Illustration of the proposed designer concept for tuning PCB X-ray radiation energy, which consists of combining two crystalline materials into a heterostructure whose periodicity is customized by the choice of layers. VdW stacking and molecular beam epitaxy provide possible approaches to these heterostructures. (b) X-ray emission peak energy of superlattice structures formed by alternating layers of graphite $\left(d_{1}=0.3308 \mathrm{~nm}\right)$ and hexagonal boron nitride $\left(d_{2}=\right.$ $0.3350 \mathrm{~nm}$ ), used as an illustrative example. Each superlattice contains a different number of 
layers from each material, as shown in the models on the right: $n_{1}=1, n_{2}=1$ (yellow); $n_{1}=$ $1, n_{2}=3$ (red); and $n_{1}=1, n_{2}=8$ (blue). The different choice of superlattice enables a direct control over the number and energy of the resulting emission peaks. The choice of different emission orders $(m)$ enables coarse or fine energy tunability, as demonstrated in the plot and in the inset, respectively. (c) Full emission spectra simulated under the conditions indicated by the dashed line in (b) (300 keV kinetic energy), showing that the most dominant order of emission for each structure is $m=n_{1}+n_{2}$ (see Eq. 3). 


\section{Methods}

Experimental. The radiation was generated by highly collimated electron beams in a FEI Titan Themis G2 transmission electron microscope (TEM) with acceleration voltage in the range of $60-300 \mathrm{kV}$, which is used to control the velocity of the incident electrons. The energy spectrum was measured using a Dual Bruker XFlash6 $\mid 100$ EDS detector oriented at $\varphi=121^{\circ}$ with respect to the electron velocity vector and with angular aperture of $\Delta \varphi_{\mathrm{D}}=16^{\circ}$. The energy resolution of the detector in the energy range of the measurements is $\sim 80 \mathrm{eV}$ full-width at halfmaximum (FWHM). The resolution was determined by fitting Gaussian distribution to the characteristic radiation energy peaks of $\mathrm{W}$ and Se in proximity to the PCB radiation peaks. All spectra were obtained by aligning the collimated electron beams along the crystals zone axis ([103] and [001]), verified by their diffraction patterns. The input power in our experiment was of the order of $\sim 0.1 \mathrm{~mW}(\sim 100 \mathrm{kV}$ times $1 \mathrm{nA})$, much lower than that of commercial X-ray tubes, which lies in the range of 10 's to 1000 's W. The relatively low input power required relativity long accumulation times of 16 min during the experiment. However, no visible damage was detected on the sample after up to 10 such measurements.

Theoretical The theoretical prediction of the peak emission (vertical lines in the figures) were obtained using Eq. 1 without any fitting parameters. We developed two simulation frameworks. In the CBS simulator, the trajectories of the electrons traveling in the vdW material were obtained by solving the relativistic Newton-Lorentz equations using a fifth-order Runge Kutta algorithm [34,55]. The impinging electrons were assumed to be normally incident on the plane of the vdW layers. The atomic potential of the vdW material was obtained from density functional theory (DFT) computations that take into consideration all inner-shell electrons [56, 57]. The DFT calculations were performed with the GPAW code [58], which is based on the 
projector augmented wave method, using a plane wave basis set to expand the electron wave functions. The radiation from the electrons was calculated from their trajectories via the LiénardWiechert formula. Since the electron beam width is far larger than the transverse lattice cell size, the total radiation spectrum was obtained by averaging over the radiation spectra of individual electrons spanning one-unit cell of the atomic structure.

In the PXR simulator, the atomic structure was modelled by periodic dipole arrays and the incident electrons were assumed to travel along straight lines with uniform velocity. The response of the bound electrons around each atom was modelled by effective dipoles, with the atomic polarizability derived from the experimental scattering factor [59].

The widths of the peaks in Fig. 1 and Fig. 2 were calculated by combining the energy width $\Delta \mathrm{E}$ (calculated using Eq. 2) with the energy broadening due to the detector energy resolution $\Delta \mathrm{E}_{\mathrm{Res}}$ for an approximate energy width of $\Delta \mathrm{E}_{\mathrm{tot}}=\sqrt{\Delta \mathrm{E}^{2}+\Delta \mathrm{E}_{\mathrm{Res}}^{2}}$. The energy widths were also found in another more precise way, using the PXR simulation, and convolving the spectra with a Gaussian (FWHM of $\Delta \mathrm{E}_{\mathrm{Res}}$ ) that models the response of the detector. The results yield the theoretical spectral line presented in Fig. 1. The obtained energy widths in both methods approximates well the experimental data.

Sample Preparation. Chromium thiophosphate (CrPS4) was synthesized by vapor transport synthesis (VTS) with temperature gradient $750^{\circ} \mathrm{C} / 710^{\circ} \mathrm{C}$ and a reaction time of 4 days, as described in detail elsewhere [60]. Transition metal phosphorous trisulfides were synthesized by the same technique, but the reaction time was longer $(1$ week $)$ and the temperature gradient was different for each of the specific compound: $\mathrm{MnPS} 3: 650^{\circ} \mathrm{C} / 600^{\circ} \mathrm{C}$; FePS3: $650^{\circ} \mathrm{C} / 550^{\circ} \mathrm{C}$; CoPS3: $625^{\circ} \mathrm{C} / 575^{\circ} \mathrm{C}$; and NiPS3: $650^{\circ} \mathrm{C} / 600^{\circ} \mathrm{C}$. The $\mathrm{vdW}$ materials were mechanically exfoliated and directly transferred to a TEM grid using a protocol described in Ref. [60]. 


\section{Data and Code Availability Statements}

The data and code that supports the plots within this paper and other findings of this study are available from the corresponding author upon reasonable request

\section{Corresponding Authors}

For correspondence and additional materials please contact Michael Shentcis (Michael.shentcis@gmail.com) or Ido Kaminer (kaminer@technion.ac.il). 


\section{References}

[1] Novoselov, K. S. et al. Two-dimensional atomic crystals. Proc. Natl Acad. Sci. USA 102, 10451-10453 (2005).

[2] Basov, D. N., Fogler, M. M. and García de Abajo, F. J. Polaritons in van der Waals materials. Science 354, aag1992 (2016).

[3] Low, T., et al. Polaritons in layered two-dimensional materials. Nature Mater. 16, 182-194 (2016).

[4] Novoselov, K. S. et al. Electric field effect in atomically thin carbon films. Science 306, 666669 (2004).

[5] Castro Neto, A. H., Guinea, F., Peres, N. M. R., Novoselov, K. S. and Geim, A. K. The electronic properties of graphene. Rev. Mod. Phys. 81, 109-162 (2009).

[6] Hao, J. et al. High performance optical absorber based on a plasmonic metamaterial. Appl. Phys. Lett. 96, 251104 (2010).

[7] Lee, C., Wei, X., Kysar, J. W. and Hone, J. Measurement of the elastic properties and intrinsic strength of monolayer graphene. Science 321, 385-388 (2008).

[8] Balandin, A. A. Thermal properties of graphene and nanostructured carbon materials. Nature Mater. 10, 569-581 (2011).

[9] Kane, C. L. and Mele, E. J. Quantum spin Hall effect in graphene. Phys. Rev. Lett. 95, 226801 (2005).

[10] Wang, Q. H., Kalantar-Zadeh, K., Kis, A., Coleman, J. N. and Strano, M. S. Electronics and optoelectronics of two-dimensional transition metal dichalcogenides. Nature Nanotech. 7, 699-712 (2012).

[11] Chhowalla, M. et al. The chemistry of two-dimensional layered transition metal dichalcogenide nanosheets. Nature Chem. 5, 263-275 (2013).

[12] Evain, M., Brec, R., Wbangbo, M. H. Structural and electronic properties of transition metal thiophosphates J. Solid-state Chem. 71, 244-262 (1987).

[13] Latini, S., Olsen, T. and Thygesen, K. S. Excitons in van der Waals heterostructures: the important role of dielectric screening. Phys. Rev. B 92, 245123 (2015).

[14] Jariwala, D., Sangwan, V. K., Lauhon, L. J., Marks, T. J. and Hersam, M. C. Emerging device applications for semiconducting two-dimensional transition metal dichalcogenides. ACS Nano 8, 1102-1120 (2014).

[15] Susner, M. A., Chyasnavichyus, M., McGuire, M. A., Ganesh, P. and Maksymovych, P. Metal thio-and selenophosphates as multifunctional van der Waals layered materials. Adv. Mater. 29, 1602852 (2017).

[16] Überall, H. High-energy interference effect of Bremsstrahlung and pair production in crystals Phys. Rev. 103, 1055-1067 (1956).

[17] Korobochko, Y. S., Kosmach, V. F., and Mineev, V. I. On coherent electron bremsstrahlung. Sov. Phys. JETP 21, 834-839 (1965).

[18] Baryshevsky, V. G., and Feranchuk, I. D. Parametric X-rays from ultrarelativistic electrons in a crystal: theory and possibilities of practical utilization. J. Phys. (Paris) 44, 913-922 (1983).

[19] Baryshevsky, V. G., Feranchuk, I. D. and A. P. Ulyanenkov, Parametric X-Ray Radiation in Crystals (Springer, Berlin, Heidelberg, 2005).

[20] Jiang, P., Qian, X., Gu, X., and Yang, R. Probing Anisotropic Thermal Conductivity of Transition Metal Dichalcogenides $\mathrm{MX}_{2}(\mathrm{M}=\mathrm{Mo}, \mathrm{W}$ and $\mathrm{X}=\mathrm{S}$, Se) using Time-Domain Thermoreflectance. Adv. Mater. 29, 1701068 (2017).

[21] Zan, R., et al. Control of radiation damage in MoS2 by graphene encapsulation. ACS Nano 7, 10167-10174 (2013). 
[22] Lehnert, T., Lehtinen, O., Algara-Siller, G., and Kaiser, U. Electron radiation damage mechanisms in 2D MoSe 2 . Appl. Phys. Lett. 110, 033106 (2017).

[23] Kirz, J., Jacobsen, C. and Howells, M. Soft X-ray microscopes and their biological applications. Q. Rev. Biophys. 28, 33-130 (1995).

[24] de Groot, F. and Kotani, A. Core Level Spectroscopy of Solids (CRC Press, 2008).

[25] Hitchcock, A. P. Soft X-Ray Spectromicroscopy and Ptychography. J. Electron Spectrosc. Relat. Phenom. 200, 49-63 (2015).

[26] Agarwal, B. K. X-ray spectroscopy: an introduction. (Springer, Vol. 15, 2013).

[27] Hayakawa Y. et al. X-ray imaging using a tunable coherent X-ray source based on parametric X-ray radiation, J. Instrumentat. 8, C08001 (2013).

[28] Carroll, F. E. Tunable monochromatic X rays: a new paradigm in medicine. Am. J. Roentgenol. 179, 583-590 (2002).

[29] Okada, H., et al. Basic Study of Parametric X-ray Radiation for Clinical Diagnosis using 125MeV Linear Particle Accelerator. Journal of Hard Tissue Biology 24, 299-302 (2015).

[30] Saldin, E. L., Schneidmiller, E. A. and Yurkov, M. The Physics of Free-Electron Lasers (Springer, Berlin, 2000).

[31] Ackermann, W. et al. Operation of a free-electron laser from the extreme ultraviolet to the water window. Nature Photon. 1, 336-342 (2007).

[32] H. Winick and S. Doniach, Synchrotron Radiation Research (Springer Science and Business Media, 2012).

[33] Powers, N. D. et al. Quasi-monoenergetic and tunable X-rays from a laser-driven Compton light source. Nature Photon. 8, 28-31 (2014).

[34] Wong, L. J., Kaminer, I., Ilic, O., Joannopoulos, J. D. and Soljačió, M. Towards graphene plasmon-based free-electron infrared to X-ray sources. Nature Photon. 10, 46-52 (2016).

[35] Rosolen, G. et al. Metasurface-based multi-harmonic free-electron light source. Light Sci. Appl. 7, 64 (2018).

[36] Pizzi, A. et al. Graphene Metamaterials for Intense, Tunable, and Compact Extreme Ultraviolet and X-Ray Sources. Adv. Sci. 7, 1901609 (2019).

[37] Rivera, N., Wong, L. J., Joannopoulos, J. D., Soljačić, M., and Kaminer, I. Light emission based on nanophotonic vacuum forces. Nature Phys. advanced online publication (2019).

[38] Blazhevich, S. V. et al. First observation of interference between parametric X-ray and coherent bremsstrahlung Phys. Lett. A 195, 210-212 (1994).

[39] Feranchuk, I. D., Ulyanenkov, A., Harada, J. and Spence, J. C. H. Parametric X-ray radiation and coherent bremsstrahlung from nonrelativistic electrons in crystals. Phys. Rev. E 62, 42254234 (2000).

[40] Fraser, J. S., Sheffield, R. L., and Gray, E. R. A new high-brightness electron injector for free electron lasers driven by RF linacs. Nucl. Instrum. Methods A 250, 71-76 (1986).

[41] Dunham, B. et al. Record high-average current from a high-brightness photoinjector. Appl. Phys. Lett. 102, 034105 (2013).

[42] Li, X., et al. Dispersion engineering in metamaterials and metasurfaces J. Phys. D 51, 054002 (2018).

[43] Kaminer, I. et al. Spectrally and spatially resolved Smith-Purcell radiation in plasmonic crystals with short-range disorder. Phys. Rev. X 7, 011003 (2017).

[44] Remez, R. et al. Spectral and spatial shaping of Smith-Purcell radiation. Phys. Rev. A 96, 061801 (2017).

[45] Geim, A. K. and Grigorieva, I. V. Van der Waals heterostructures. Nature 499, 419-425 (2013).

[46] Gjerding, M. N., Petersen, R., Pedersen, T. G., Mortensen, N. A. and Thygesen, K. S. Layered van der Waals crystals with hyperbolic light dispersion. Nature Commun. 8, 320 (2017). 
[47] Herman, M. A. and Sitter, H. Molecular Beam Epitaxy: Fundamentals and Current Status (Springer Science \& Business Media, 2012).

[48] Dapkus, P. D. Metalorganic chemical vapor deposition. Annu. Rev. Mater. Sci. 12, 243-269 (1982).

[49] Graves, W., Kärtner, F., Moncton, D. and Piot, P. Intense superradiant x rays from a compact source using a nanocathode array and emittance exchange. Phys. Rev. Lett. 108, 263904 (2012).

[50] Nanni, E. A., Graves, W. S. \& Moncton, D. E. Nanomodulated electron beams via electron diffraction and emittance exchange for coherent X-ray generation. Phys. Rev. Accel. Beams 21, 014401 (2018).

[51] Naumova, N. et al. Attosecond electron bunches. Phys. Rev. Lett. 93, 195003 (2004).

[52] Lim, J., Chong, Y. and Wong, L. J. Terahertz-optical intensity grating for creating highcharge, attosecond electron bunches. New Journal of Physics 21, 033020 (2019).

[53] Attwood, D. T. Soft X-Rays and Extreme Ultraviolet Radiation (Cambridge Univ. Press, Cambridge, UK, 2000.

[54] Roessl, E. et al. Sensitivity of photon-counting based K-edge imaging in X-ray computed tomography. IEEE Trans. Med. Imaging 30, 1678-1690 (2011).

[55] Wong, L. J. et al. Laser-induced linear-field particle acceleration in free space. Sci. Rep. 7, 11159 (2017).

[56] Wang, W. L., and Kaxiras, E. Efficient calculation of the effective single-particle potential and its application in electron microscopy. Phys. Rev. B 87, 085103 (2013).

[57] Susi, T. et al. Efficient first principles simulation of electron scattering factors for transmission electron microscopy. Ultramicroscopy 197, 16-22 (2019).

[58] Enkovaara, J. et al. Electronic structure calculations with GPAW: A real-space implementation of the projector augmented wave method. J. Phys. Condens. Matter 22, 253202 (2010).

[59] Henke, B. L., Gullikson, E. M. and Davis, J. C. X-ray interactions: photoabsorption, scattering, transmission, and reflection at $\mathrm{E}=50-30000 \mathrm{eV}, \mathrm{Z}=1-92$. At. Data Nucl. Data Tables 54, 181-342 (1993).

[60] Budniak, A. K. et al. Exfoliated $\mathrm{CrPS}_{4}$ with Promising Photoconductivity. Small 16, 1905924 (2020). 


\section{Acknowledgments}

We thank Dr. Yaron Kauffman for his advice and the fruitful discussion. This work was supported by the ERC (Starter Grant No. 851780) and the ISF (Grant No. 830/19). This work was also supported by the European Commission via the Marie-Sklodowska Curie action Phonsi (H2020-MSCA-ITN-642656). H.H.S also acknowledges the support of Marie-Sklodowska Curie action Phonsi (H2020-MSCA-IF-2018-843830). L.J.W. acknowledges the support of the Agency for Science, Technology and Research (A*STAR) Advanced Manufacturing and Engineering Young Individual Research Grant (A1984c0043), and the Nanyang Assistant Professorship Startup Grant. F.J.G.A acknowledges support from the Spanish MINECO (Grants No. MAT201788492-R and No. SEV2015-0522), ERC (Advanced Grant No. 789104-eNANO), the Catalan CERCA Program, and Fundació Privada Cellex. I.K. was also supported by the Azrieli Faculty Fellowship.

\section{Statement of author contribution}

M.S. spearheaded the project, designed and performed the electron microscopy experiments, prepared the samples, analyzed the data, and developed the superlattice theory. A.K.B. contributed to the measurements and performed electron microscopy experiments. A.K.B., H.H.S., M.B., Y.A., S.T., F.H.L.K., and E.L. created the van der Waals materials. R.D. and M.K. advised on experimental aspects. X.S., Y.K., and F.J.G.A., developed and executed the PXR simulations. M.K.S. and K.S.T. performed the density functional theory simulations. L.J.W. developed and executed the CBS simulations. M.S. and I.K. conceived the idea. I.K. supervised the project. 